\title{
Sustained Ventricular Fusion Simulating a Biventricular Pacing
}

\author{
Vincenzo Carbone ${ }^{1, *}$, Vincenzo Marafioti ${ }^{2}$, Maria Pia Calabro ${ }^{3}$, \\ Giuseppe Oreto ${ }^{4}$
}

${ }^{1}$ Department of Clinical and Experimental Medicine, University of Messina, Messina, Italy

${ }^{2}$ Cardiovascular and Thoracic Department, University Hospital of Verona, Verona, Italy

${ }^{3}$ Department of Pediatrics, University of Messina, Messina, Italy

${ }^{4}$ Department of Clinical and Experimental Medicine, University of Messina,

Messina, Italy

${ }^{*}$ Corresponding author: Vincenzo Carbone, Department of Clinical and Experimental Medicine, University of Messina, via Europa, Giuseppe Vesuviano (Naples),

DOI: $10.21859 / \mathrm{ijcp}-020104$ Messina, Italy. Tel: +39-0815287237, E-mail: carbovincenzo61@gmail.com

Submited: 11.28 .2016

Accepted: 05.01.2017

\section{Keywords:}

Pacemaker, Artificial

Heart Ventricles

Electrocardiography

(C) 2016. International Journal of Cardiovascular Practice.

\begin{abstract}
An electrocardiogram from a patient implanted with a dual-chamber DDD pacemaker showed paced QRS complexes whose morphology and frontal-plane axis were consistent with biventricular stimulation with right ventricular lead located at the apex. However, some electrocardiographic findings were suggestive, rather, of univentricular right apical pacing and sustained ventricular fusion with competing native atrioventricular conduction in the presence of patient's spontaneous QRS showing right bundle branch block plus left anterior hemiblock. Shortening atrioventricular delay with magnet application advanced right ventricle stimulation and prevented the supraventricular impulse to contribute to ventricular depolarization, thereby making clear the mechanism of right ventricular apical pacing.
\end{abstract}

\section{CASE PRESENTATION}

A 67-year-old male with chronic heart failure underwent dual-chamber DDD pacemaker implantation for recurrent syncope due to paroxysmal third-degree atrioventricular (AV) block. The main setting parameters of the pulse generator were as follows: lower rate $=70 \mathrm{ppm}$; paced atrioventricular interval $(\mathrm{pAVI})=160 \mathrm{~ms}$

The Fig 1 shows the Electrocardiogram (ECG) recorded from the same patient two years later.

What about the ventricular pacing site(s).

\section{DISCUSSION}

The paced QRS complexes are not typical for right ventricular (RV) pacing from the apex, since: 1) negative complexes are present not only in leads II, III and aVF, but also in lead I, whereas aVL shows isodiphasic complexes; 2) the frontal plane QRS axis is directed at about -120 degrees; and 3) the paced QRS complexes are relatively narrow, their duration being about $100 \mathrm{~ms}$. The above data suggest, at first glance, a biventricular pacing, for both the right superior QRS axis direction with relatively narrow ventricular complexes, and the right bundle branch block (RBBB)-like morphology (dominant $\mathrm{R}$ wave) of the paced beats in lead V1 [1]. Such a pattern, indeed, is commonly seen during biventricular pacing when the RV lead is located at the apex, particularly if the $\mathrm{V}-\mathrm{V}$ interval is set in order to make left ventricle (LV) stimulation ahead of RV stimulation $[1,2]$. Some findings, however, are quite inconsistent with this interpretation. First, pAVI timing at $160 \mathrm{~ms}$ is too long to allow maximal hemodynamic response to Cardiac Resynchronization Therapy (CRT). Furthermore, AVI optimization procedure in CRT patients usually leads to values in the range of 80 to $100 \mathrm{~ms}$ [3]. Second, the terminal $r$ wave in lead III suggests, rather, an RBBB. Third, the paced QRS morphology in lead V1 (rR') rules out biventricular pacing with RV lead in the apex, because in this setting the ECG shows a pattern of initial dominant $R$ wave $[1,2]$, whereas $r R^{\prime}$ configuration is typical for RBBB. Fig 2 shows a strip of ECG recorded during magnet application. In the magnet mode, the pacing rate is increased to 100 per minute and the pAVI is decreased to $100 \mathrm{~ms}$. The pacemaker-induced QRS complexes now show a typical configuration of RV apical pacing: left superior axis deviation, expressed by QRS which are negative in the inferior leads (more negative in lead III than in lead II) and predominantly positive in lead I; increased QRS duration to 140 ms [1-3]. It is thus clear that the QRS complexes shown in Figure 1 are ventricular fusion beats with spontaneously 
conducted QRS during conventional RV apical pacing. The fusion mechanism occurs since the intrinsic AV conduction time is almost identical to the programmed AVI, so that ventricular activation results from simultaneous invasion of the ventricles by supraventricular (paced atrial) and ectopic (paced) ventricular impulses. The patient's spontaneous ECG showed RBBB plus left anterior hemiblock and a P-R interval of about $150 \mathrm{~ms}$. In the presence of a bundle branch block, the ventricular fusion due to end-diastolic ectopic impulses arising in the ipsilateral ventricle to the impaired bundle branch can result in reduction or nullification of the effect of the block. This, thereby, leads to "normalized" QRS complexes [4]. Likewise, in the reported case, the fusion phenomenon resulting from RV pacing and simultaneous anterograde propagation of the impulse of atrial origin counterbalances in part the native conduction disturbance [5]. On the other hand, stimulation of the RV from the apex strengthens the frontal plane left axis deviation caused by the left anterior hemiblock. During DDD pacing (Figure 1), the LV was mainly depolarized by the paced atrial impulse conducted over the His bundle and the posterior division of left bundle branch, whereas the RV was activated by the artificial impulse [5]. In the magnet mode (DOO), instead, the shortened AV delay advances RV stimulation, so that the impulse coming from the atria is prevented to play any role in ventricular depolarization and a full pacemaker-induced ventricular capture occurs without fusion. Thus, depolarization of the 2 ventricles during AV sequential pacing in DDD mode was more synchronous than both in sinus rhythm and in
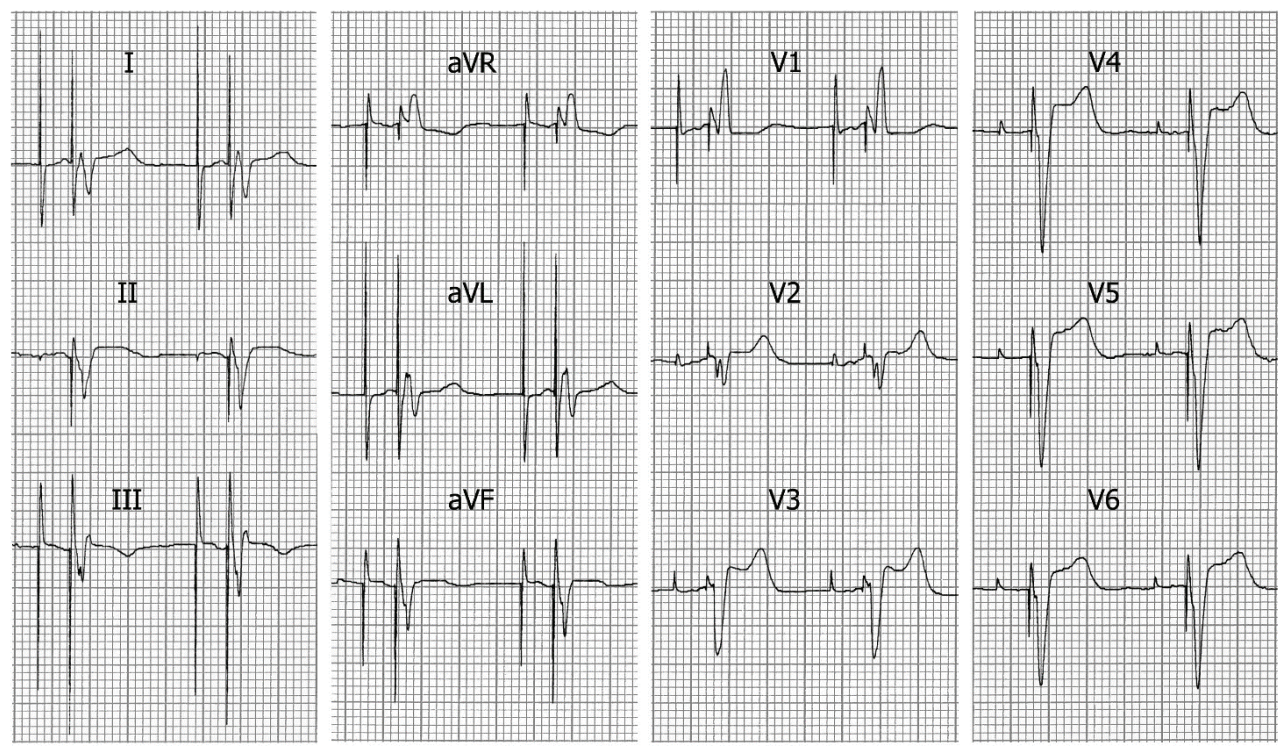

Figure 1: 12-lead ECG

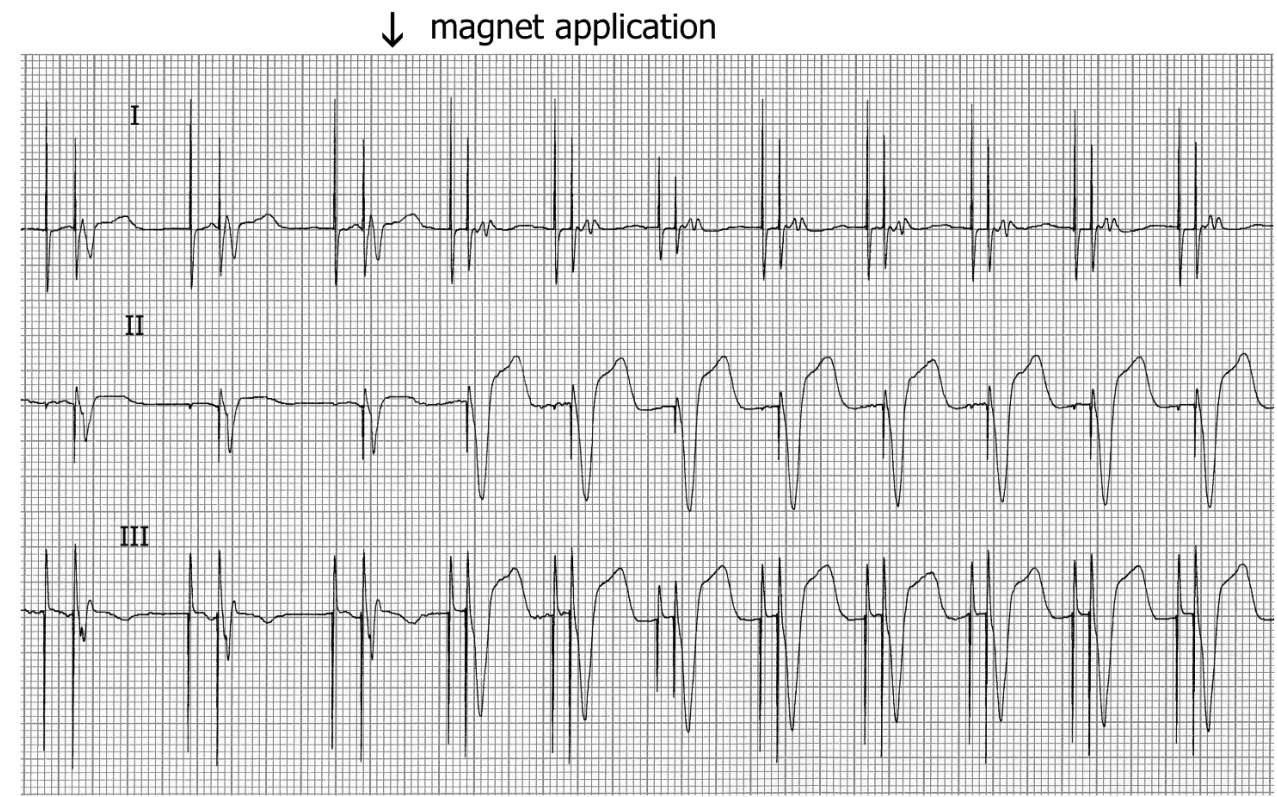

Figure 2: ECG leads I, II and III Recorded During Magnet Application 
magnet mode pacing, thereby resulting in relatively narrow QRS complexes with a configuration that, at first glance, suggested biventricular, rather than RV, stimulation [5]. This case reveals that the ECG presentation of cardiac pacing is sometimes misleading and the diagnosis of biventricular stimulation based on current criteria [1] should be only entertained after excluding ventricular fusion between paced univentricular activation and competing native AV conduction. This represents a possible pitfall whenever the $\mathrm{P}-\mathrm{R}$ interval is nearly identical to the programmed AV delay $[4,5]$. Ventricular fusion is ascertained or ruled out by observing the paced QRS morphology during AVI shortening [1-3]. In the presence of a relatively long programmed AVI, the response to magnet application can help us address this issue simply and quickly.

\section{FUNDING}

Authors declared there is no funding or support.

\section{REFERENCES}

1. Barold SS, Stroobandt RX, Sinnaeve AF. Cardiac pacemakers and resynchronization step by step: An illustrated guide. Oxford: John Wiley \& Sons; 2010.

2. Barold SS, Herweg B. Usefulness of the 12-lead electrocardiogram in the follow-up of patients with cardiac resynchronization devices. Part I. Cardiol J. 2011;18(5):476-86. PMID: 21947982

3. Sweeney MO. Cardiac resynchronization therapy. In: Ellenbogen KA, Wood MA, editors. Cardiac Pacing and ICDs. 4th ed. Oxford: Blackwell; 2005. p. 415-66.

4. Schamroth L. The disorders of cardiac rhythm. 2nd ed. Oxford: Wiley-Blackwell; 1971

5. Oreto G, Luzza F, Donato A, Carbone V, Satullo G, Calabrò MP. Bar: in mosaic tiles 12. Milano: Edi-Ermes; 2009. 